\title{
Measurement of High Energy Neutrons via Lu(n,xn) Reactions
}

\author{
E. A. Henry, J. A. Becker, D. E. Archer, W. Younes \\ M. A. Stoyer, and D. Slaughter \\ Lawrence Livermore National Laboratory \\ Livermore, CA 94550
}

This paper was prepared for submittal to the

American Nuclear Society

Albuquerque, NM

November 16-20, 1997

July, 1997

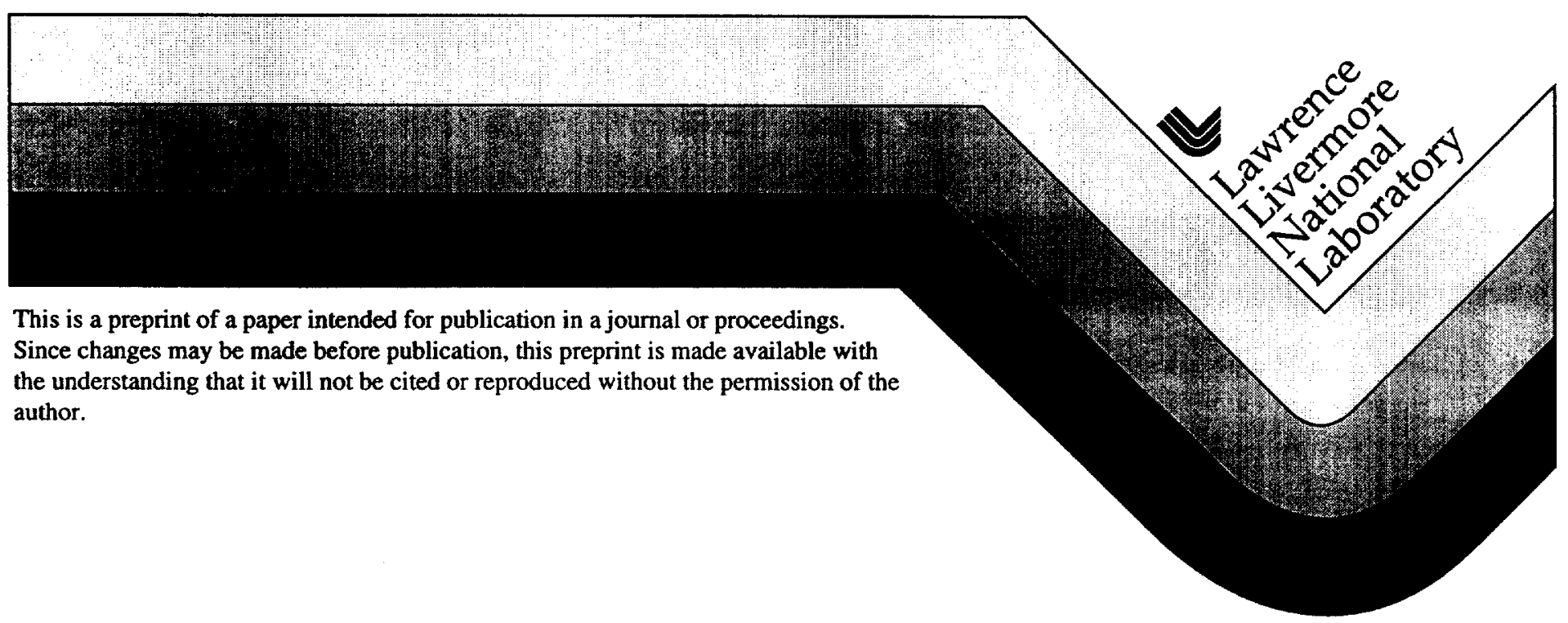




\section{DISCLAMMER}

This document was prepared as an account of work sponsored by an agency of the United States Government. Neither the United States Govemment nor the University of California nor any of their employees, makes any warranty, express or implied, or assumes any legal liability or responsibility for the accuracy, completeness, or usefulness of any information, apparatus, product, or process disclosed, or represents that its use would not infringe privately owned rights. Reference herein to any specific commercial product, process, or service by trade name, trademark, manufacturer, or otherwise, does not necessarily constitute or imply its endorsement, recommendation, or favoring by the United States Government or the University of California. The views and opinions of authors expressed herein do not necessarily state or reflect those of the United States Government or the University of California, and shall not be used for advertising or product endorsement purposes. 


\title{
Measurement of High Energy Neutrons via $L u(n, x n)$ Reactions
}

\author{
E. A. Henry, J. A. Becker, D. E. Archer, W. Younes, M. A. Stoyer, and D. Slaughter \\ Lawrence Livermore National Laboratory \\ Livermore, CA 94550 \\ (510) $-422-5532$
}

\section{ABSTRACT}

High energy neutrons can be assayed by the use of the nuclear diagnostic material lutetium. We are measuring the $(n, x n)$ cross sections for natural lutetium in order to develop it as a detector material. We are applying lutetium to diagnose the high energy neutrons produced in test target/blanket systems appropriate for the Accelerator Production of Tritium Project.

\section{INTRODUCTION}

We are developing lutetium $(\mathrm{Lu})$ as a detector material to measure the spectrum and flux of high energy neutrons. Highpowered spallation sources are being designed to produce large amounts of energetic neutrons for applications and for use in basic science. The Accelerator Production of Tritium (APT) Project is one application that envisions the use of a highcurrent, high-energy proton beam bombarding a target/blanket system to generate copious amounts of neutrons. These neutrons are thermalized and captured on ${ }^{3} \mathrm{He}$; producing tritium. The tritium is then extracted and purified for national needs.

A series of demonstration tests have been conducted to measure tritons produced per incident proton, and neutrons produced per incident proton, in target/blanket configurations appropriate to APT. These tests are being modeled by the LAHET/MCNP (LCS) code system, the same code system being used to design and model the APT production target. Nuclear activation data from a variety of materials are being used in these demonstrations to test and constrain the LCS, providing confidence in the modeling and design of the production target. Activation materials employed, such as $\mathrm{Au}, \mathrm{Mn}$, and ${ }^{3} \mathrm{He}$, are typically responsive to low energy neutrons. While some $(n, 2 n)$ activation materials are available, these are generally used only to assay neutrons that have a 14$\mathrm{MeV}$ spectrum. Lu offers the advantage that neutrons with energies to about 100 $\mathrm{MeV}$ can be assayed, with the aim of providing additional tests and constraints on calculated high energy neutron spectra.

In this paper we discuss the advantages of $\mathrm{Lu}$ as a high energy neutron detector, including nuclear data, production of target foils, and gamma-ray spectrometry to deduce the amount of activation products. $\mathrm{Lu}(\mathrm{n}, \mathrm{xn})$ cross sections are being measured. for neutron energies up to $800 \mathrm{MeV}$ with the GEANIE spectrometer at LANSCE. Use of $\mathrm{Lu}$ in an APT demonstration test is described, and some preliminary measured results are presented. 


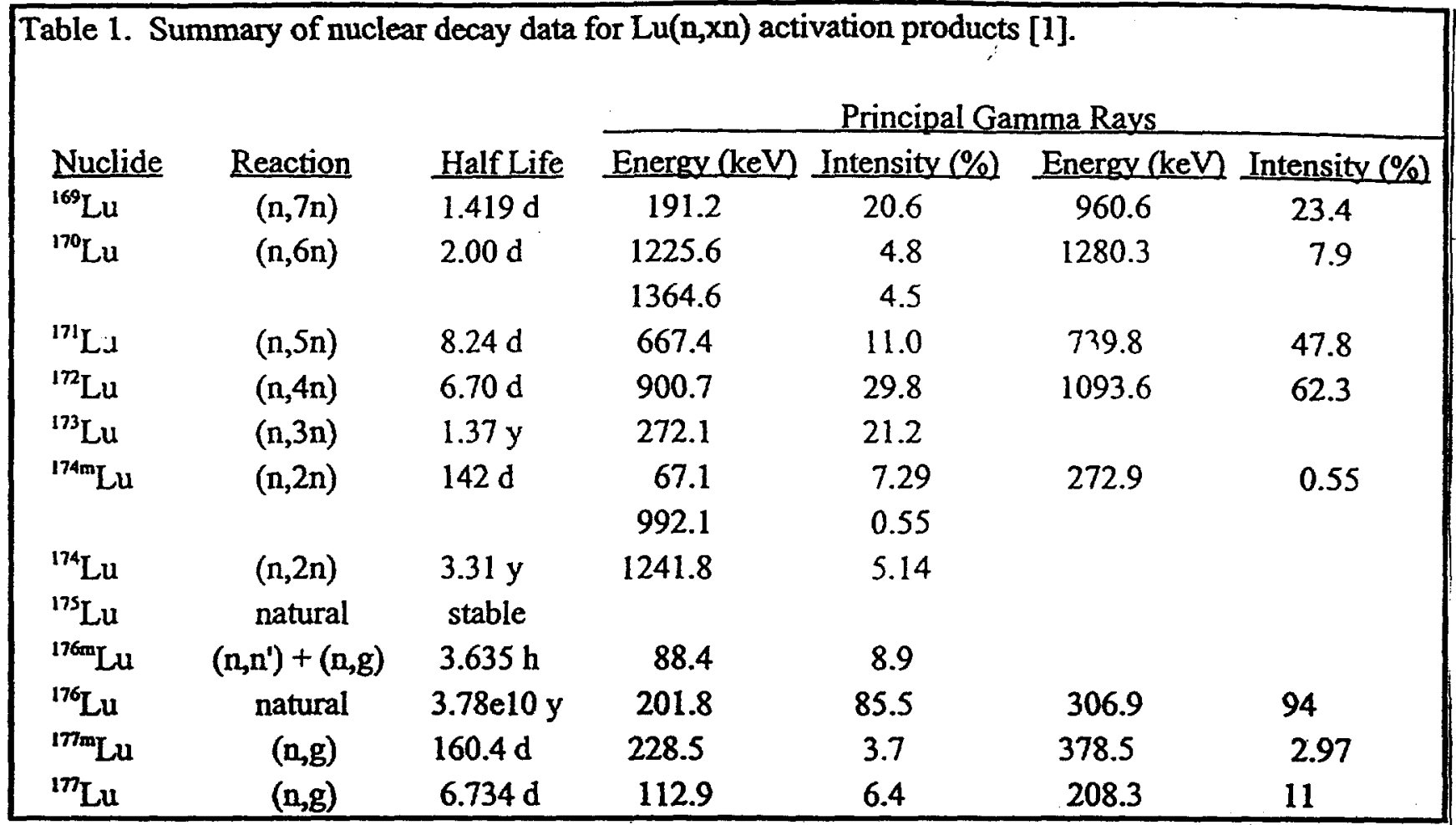

\section{RELEVANT NUCLEAR DATA FOR LU}

$\mathrm{Lu}$ is nearly mono-isotopic, with ${ }^{175} \mathrm{Lu}$ constituting 97.41 atom percent of natural $\mathrm{Lu}$; the small remaining fraction is ${ }^{176} \mathrm{Lu}$. Thus activation products formed by high energy $(n, x n)$ reactions arise principally from ${ }^{175} \mathrm{Lu}$. ${ }^{176} \mathrm{Lu}$ has a long-lived natural radioactivity that does not generally interfere with the gamma-ray measurement of other activities.

Table 1 summarizes the production. reactions and half lives of the Lu activation products, as well as the energies and intensities of the principle gamma rays that can be used to determine the amount of each product. In addition to the $(n, x n)$ products that are formed, the $(n, \gamma)$ and $\left(n, n^{\prime}\right)$ products are included as they can be measured in gamma-ray spectra. In particular, ${ }^{177} \mathrm{Lu}$ is produced by low energy neutrons. However, because ${ }^{176} \mathrm{Lu}$ has a resonance near $10^{-7} \mathrm{eV}$, it is sensitive to a somewhat different part of the neutron spectrum than detector materials such as Au. The $\left(n, n^{\prime}\right)+(n, \gamma)$ product, ${ }^{176 m} \mathrm{Lu}$, is short lived, but can be measured. It provides information on neutrons in the range of 1 to $10 \mathrm{MeV}$ as well as low energy neutrons. The high-energy neutron activation products ${ }^{168} \mathrm{Lu}$ and ${ }^{167} \mathrm{Lu}$ have half lives less than one hour long.

\section{MEASUREMENT OF LU ACTIVITIES}

Most of the gamma rays of interest for determining the production of the $L u(n, x n)$ products are above two hundred $\mathrm{keV}$, and the short-lived activities are easily observed. Figure 1 shows a spectrum of gamma rays that follow the beta decay of Lu activities produced in a APT irradiation. This spectrum shows gamma rays from 


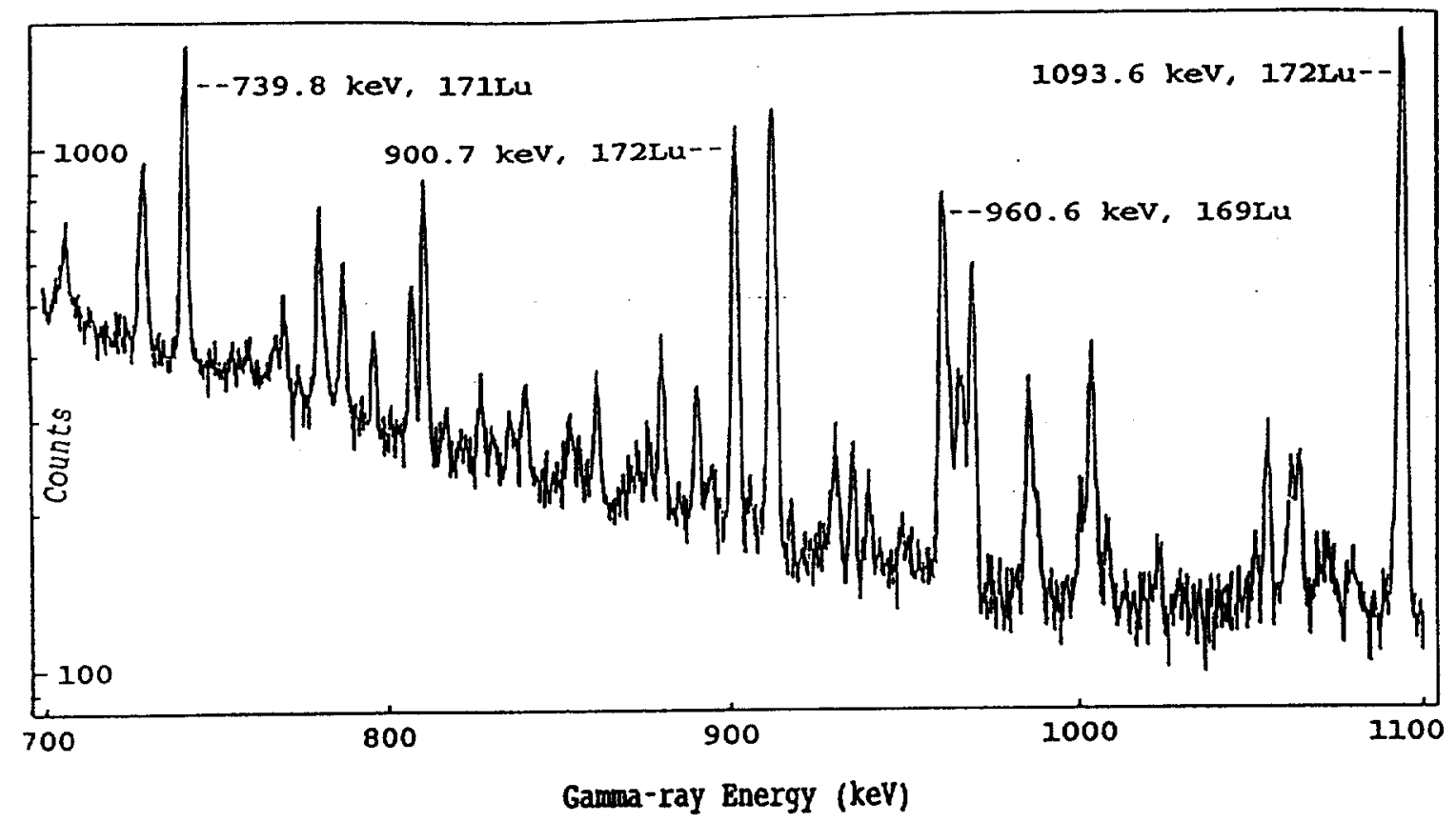

Figure 1. A spectrum of the gamma rays from a target irradiated in an APT test. The principal gamma rays from ${ }^{169,171,172} \mathrm{Lu}$ are identified. Numerous other weaker gamma rays from Lu activation, as well as some background gamma rays, are evident.

several of the $L u(n, x n)$ reaction channels.

The long-lived activities require longer counting times, and ${ }^{174 \mathrm{~m}} \mathrm{Lu}$ has a principle gamma ray which are quite low in energy, $67 \mathrm{keV}$. However, with a large $(70 \%$ efficient), shielded germanium detector with a beryllium window, it is possible to quantify almost all the $\mathrm{Lu}$ activities expected in a target exposed to a spallation neutron spectrum. The common exception is ${ }^{174 \mathrm{~m}} \mathrm{Lu}$.

The measurement of ${ }^{176 m} \mathrm{Lu}$ requires counting within a day of irradiation. While the $88-\mathrm{keV}$ gamma ray is very intense, as the number of counts in that peak decrease, it becomes difficult to observe because of other low energy gamma rays and lead $x$ rays that are typically present from shielding, if the lead is not lined with $x$-ray filters. In addition, a strong beta group is present due to the ${ }^{176 \mathrm{~m}} \mathrm{Lu}$ decay, and can add significantly to the low energy background and the total count rate. A plastic beta filter can be used in front of the thin-windowed germanium detector to reduce the efficiency of the detector for the beta rays while reducing the efficiency for detection of gamma rays only modestly.

The measurement of ${ }^{169-172,177} \mathrm{Lu}$ is best carried out one to three days after an irradiation, though the ${ }^{171,172} \mathrm{Lu}$ activities can counted for up to two weeks, depending on the source strength of each activity. The neutron capture product ${ }^{177} \mathrm{Lu}$ has the highest activity, and can be counted for many weeks after irradiation. The longer-lived activities $173,174,174 \mathrm{~m}, 177 \mathrm{~m}_{\mathrm{Lu}}$ can best be counted about six months after an ir-radiation. Of course, ${ }^{176} \mathrm{Lu}$, being a natural long-lived constituent of $\mathrm{Lu}$, can always be counted, and can serve to check 


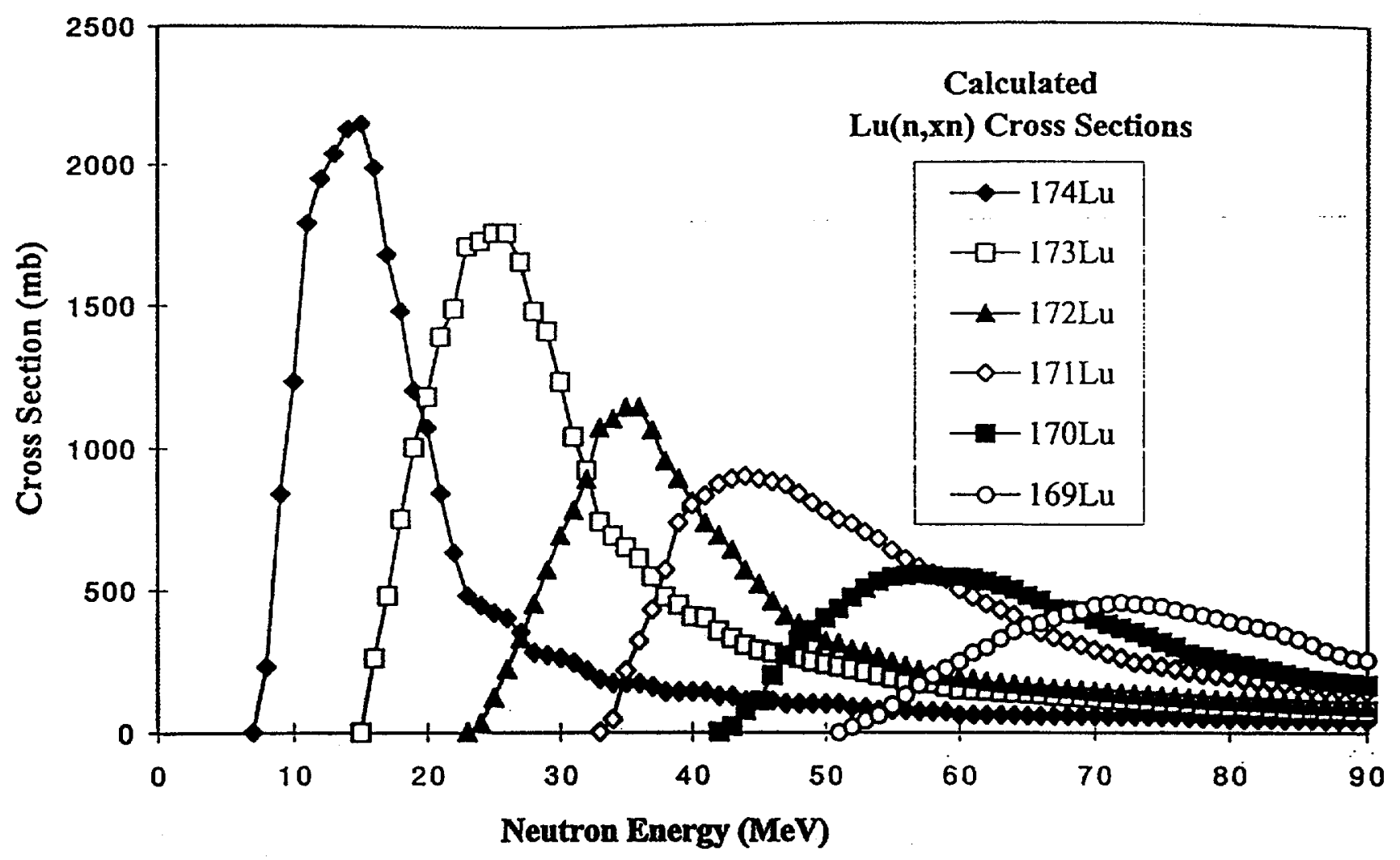

Figure 2. A plot of calculated $L u(n, x n)$ cross sections versus neutron energy. These cross sections were calculated with the ALICE code, and are being used for planning purposes.

all counting procedures and corrections that are applied, since the fraction of natural $\mathrm{Lu}$ that is ${ }^{176} \mathrm{Lu}$ is well known.

It is a good practice to determine the amount of each activation product from several gamma rays if possible. If discrepancies occur between the determinations from different gamma-rays, data can be scrutinized for interferences, the detector efficiency can be reviewed, the target self absorption can be checked, and other sources of error can be sought.

\section{LU(N,XN) CROSS SECTIONS}

Interpretation of the measured number of atoms of each species to determine the neutron spectrum and flux requires that the reaction cross sections be known. Unfortunately, complete cross sections for high energy neutron reactions on $\mathrm{Lu}$ have not been measured. An accurate cross section for the $(n, 2 n)$ reaction has been measured at $14 \mathrm{MeV}$, and the cross sections for $(n, 2 n)$ and $(n, 3 n)$ reactions have been measured up to $28 \mathrm{MeV}^{2,3}$. However, cross sections at still higher energies have to be measured to develop $\mathrm{Lu}$ as a detector material for APT. We have calculated $\mathrm{Lu}(\mathrm{n}, \mathrm{xn})$ cross sections with the ALICE code to facilitate planning of tests and experiments (Figure 2).

We have begun measurement of the high energy neutron $(n, x n)$ reactions with the GEANIE spectrometer at LANSCE at the Los Alamos National Laboratory. LANSCE uses a high current of $800-\mathrm{MeV}$ protons bombarding a tungsten target to produce high energy neutrons. A highly collimated beam of these neutrons irradiate 


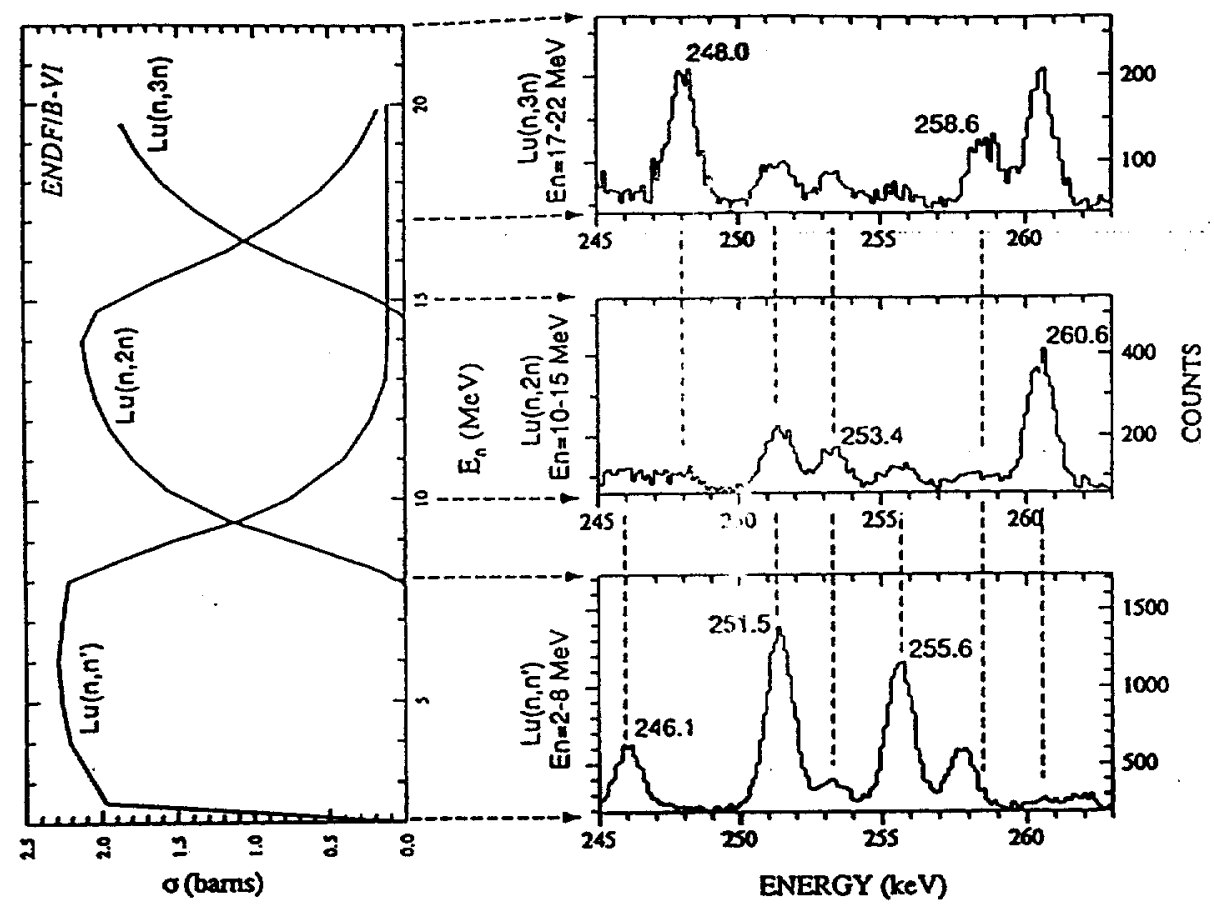

Figure 3. Preliminary data from the GEANIE spectiometer at LANSCE for $\operatorname{Lu}(n, x n)$ reactions. On the left are plots of $L u(n, x n)$ cross sections from the ENDF/B-VI library. On the right are small portions of gamma-ray spectra obtained by gating on neutron energies corresponding to the main peaks in the reaction cross sections. As can be seen, gamma rays resulting from the various channels are enhanced in the different neutron gates.

a target positioned 20 meters from the target. The GEANIE spectrometer, a detector array consisting of seven planar and thirteen coaxial germanium detectors, measures the prompt gamma rays from the neutron induced reactions. The energy of the neutron inducing the reaction is determined by its time of flight from the production target to the target material under investigation. A fission detector is used to determine the neutron flux in the beam.

We will determine the partial gammaray cross sections for each reaction channel from the GEANIE measurements, making appropriate corrections for the spectrometer efficiency, target self absorption, gammaray angular distributions, and system dead time. In principle, this method can measure the total neutron cross section, except for the cross section for direct population of the ground state or long-lived isomers. In order to determine the total cross section from these data, the partial gamma-ray cross sections will be modeled with a code such as GNASH, and the model will be then used to supplement the measured cross sections with the cross section for direct population of the ground state, long-lived isomers, and to account for weak gamma rays to the ground state missed by GEANIE, thus determining the total cross section.

Figure 3 shows some preliminary data from the GEANIE spectrometer measuring gamma rays from a Lu target. Shown on the left are cross sections for the $\left(n, n^{\prime}\right)$; $(n, 2 n)$, and $(n, 3 n)$ reactions from the ENDF/B-VI library. On the right are shown small portion of the gamma-ray 


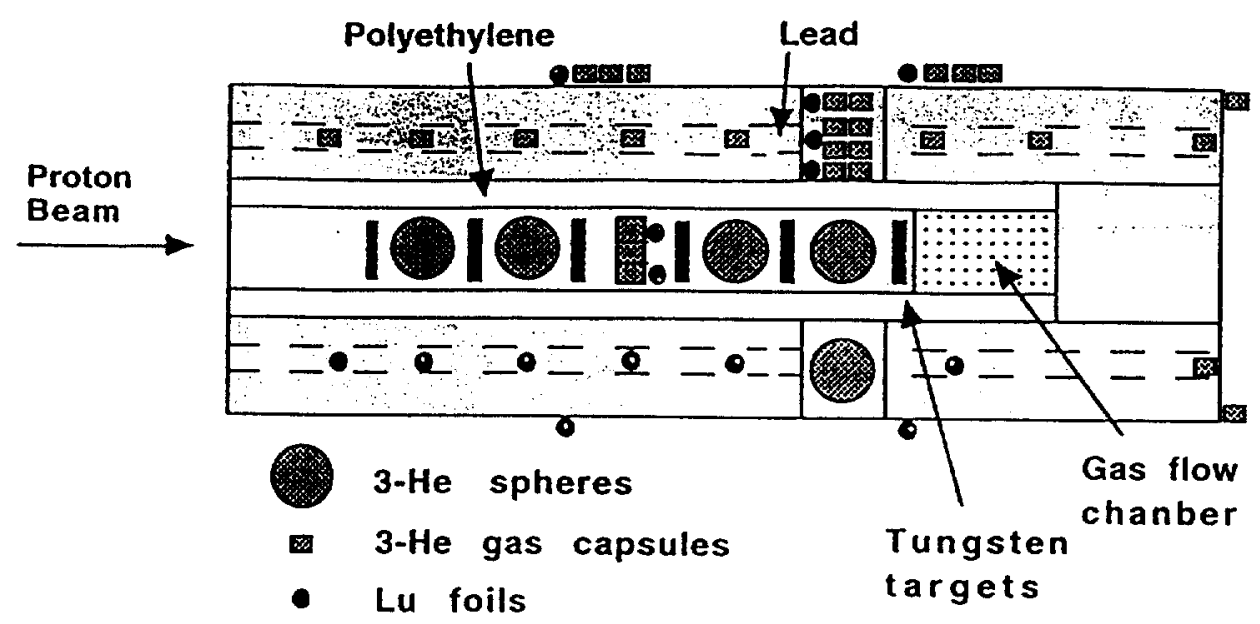

Figure 4. A schematic drawing of a target/blanket test assembly for APT, showing the ${ }^{3} \mathrm{He}$ and Lu diagnostic loadings.

spectra obtained by setting gates on time-offlight spectra corresponding to neutron energies in the main peaks of the reaction cross sections. Gamma rays from these reaction channels are enhanced in the appropriate neutron gates, while gamma rays from other channels are suppressed. These data indicate that the partial gamma-ray cross sections, and hence the total neutron reaction cross section necessary for diagnostic interpretation, can be determined.

\section{LU TARGETS}

Targets of metallic Lu can be purchased commercially; however, Lu oxidizes in air and eventually deteriorates unless carefully protected. A viable alternative is to mix $\mathrm{Lu}$ oxide into a plastic matrix and form it into sheets. We have mixed $\mathrm{Lu}_{2} \mathrm{O}_{3}$ in VCE (ethylene vinyl-acetate vinyl-alchohol terpolymer), which has carbon, oxygen, hydrogen, and nitrogen as its main constituents and can be cured to form a stable material. [The light elements in the -VCE have no long-lived activation products to interfere with the assay of $\mathrm{Lu}$ activations.] This mixture can be made with a bulk density as high as $3.5 \mathrm{gm} / \mathrm{cm}^{3}$, with $70 \%$ by weight being Lu. The plastic can be laid in thin sheets and cured to make a uniform, flexible, durable foil that can be cut with a knife. A sample $2.6 \mathrm{~cm}$ on a side and $0.1 \mathrm{~cm}$ thick contains about $1.2 \mathrm{gm}$ of $\mathrm{Lu}$, a sample size that is adequate for APT diagnostic applications.

\section{APPLICATION OF LU DIAGNOSTICS TO APT}

We have loaded Lu foils in a test of designed to measure tritium production in a target/blanket system that simulates aspects of the APT production target/blanket. The target/blanket in the test consisted of tungsten target disks; with a polyethylene thermalizer and a lead blanket. A number of ${ }^{3} \mathrm{He}$-loaded spheres and smaller ${ }^{3} \mathrm{He}$-filled capsules were located in the target/blanket, along with a number of Lu foils. Figure 4 shows a schematic drawing of the test target/blanket with the diagnostic loadings. 


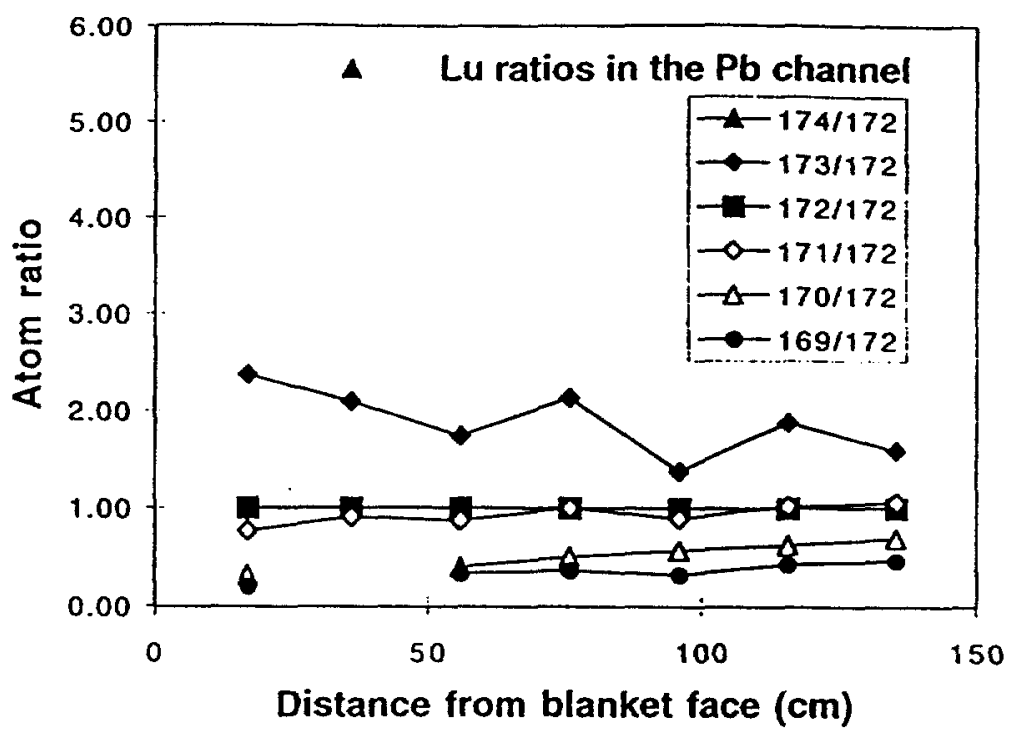

Figure 5. A plot of ratios of atoms for the different $\mathrm{Lu}$ activation products versus the distance of the diagnostic target from the front of the test assembly. These targets were loaded in a hole through the lead blanket. The ratios are normalized to ${ }^{172} \mathrm{Lu}$ production. Typical uncertainties for these ratios are $\pm 10 \%$.

After the irradiation the $\mathrm{Lu}$ foils were counted and the production of the $(n, x n)$ activation products determined. The ratios of the $\mathrm{Lu}$ activation products reflect changes in the high energy neutron spectrum as a function of $\mathrm{Lu}$ foil location in the target/blanket assembly. Figure 5 shows these ratios for the foils located in a channel in the lead blanket, normalized to the value for ${ }^{172} \mathrm{Lu}$. If the shape of the neutron spectrum is unchanged throughout the assembly, these ratios should be constant. - As can be seen, the ratios vary, and indicate that the neutron spectrum is harder as the distance from the front of the assembly increases; i.e. the production of ${ }^{171} \mathrm{Lu},{ }^{170} \mathrm{Lu}$, and ${ }^{169} \mathrm{Lu}$ increases while ${ }^{173} \mathrm{Lu}$ decreases with distance from the front of the assembly. The high value for ${ }^{174} \mathrm{Lu}$ reflects the rapid increase in neutron flux as the neutron energy decreases. Modeling of this assembly with LCS has begun, and these data will aid in testing, constraining, and validating LCS for APT applications.

\section{ACKNOWLEDGEMENTS}

This work was performed under the auspices of the U.S. Department of Energy by the Lawrence Livermore National Laboratory under Contract No. W-7405ENG-48.

\section{REFERENCES}

1. Table of Isotopes, eighth edition, Richard B. Firestone, ed., John Wiley \& Sons, Inc., New York (1996).

2. B. P. BAYHURST, J. S. GILMORE, R. J. PRESTWOOD, J. B. WILHELMY, NELSON JARMIE, B. H. ERKKILA, AND R. A. HARDEKOPF, "Cross sections for $(n, x n)$ reactions between 7.5 and $28 \mathrm{MeV}$," Physical Review C 12, 451 (1975).

3. L. R. VEESER, E. D. ARTHUR, AND P. G. YOUNG, "Cross sections for $(n, 2 n)$ and $(n, 3 n)$ reactions above $14 \mathrm{MeV}$," Physical Review C 16, 1792 (1977). 


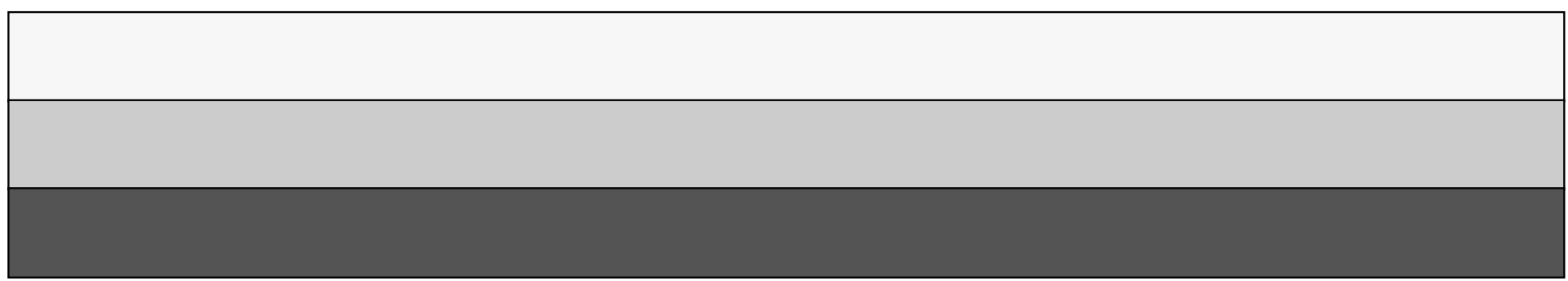

